\title{
Study on the Application of System Dynamics in the Risk Assessment of PPP Project of Expressway
}

\author{
Zhao Yongjiao \\ School of Shipping Economics and management, Dalian Maritime University, Dalian, Liaoning (125300) \\ 1040390164@qq.com
}

\begin{abstract}
:
This paper uses the method of system dynamics to study the risk factors of PPP project. First of all, on the basis of reading the relevant literature, identify the existing risks of highway PPP project, and establish the stock flow chart of highway PPP project risks. Then, the system dynamics model is used to simulate the project risk. The results show that the operation risk, completion risk and construction risk have a greater impact on the whole PPP project risk system; secondly, the key risk factors affecting the operation risk, completion risk and construction risk are analyzed. Finally, in view of the risk problems existing in the PPP project of expressway, countermeasures and suggestions are put forward. All participants of the project should pay attention to and prevent the risk, reduce the negative impact of the risk, so as to obtain the expected benefits.
\end{abstract}

Keywords: system dynamics, sensitivity analysis, PPP project

\section{系统动力学在高速公路 PPP 项目风险评价的应用研究}

\author{
赵永娇
}

\author{
大连海事大学工商管理系, 大连, 辽宁 \\ 1040390164@qq.com
}

\section{摘要:}

本文运用系统动力学的方法对影响高速公路 PPP 项目的风险因素进行研究。首先在对相关文献进行阅 读的基础上，识别了高速公路 PPP 项目存在的风险，建立了高速公路 PPP 项目风险的存量流量图。然 后, 运用系统动力学模型对项目风险进行模拟仿真。结果表明运营风险、完工风险、建设风险对整个 高速公路 PPP 项目风险系统产生的影响更大；其次，对影响运营风险、完工风险、建设风险的关键风 险因素进行分析。最后，针对高速公路 PPP 项目存在的风险问题提出了对策建议，项目各个参与方应 注意和防范风险, 减少风险带来的负面影响, 从而获得预期效益。

关键词: 系统动力学; 灵敏度分析; PPP 项目

\section{1.引言}

随着我国综合国力的提升和国家经济的不断持续 发展, 为民众提供更好的生活环境、加快城市化进程, 对基础设施的建设也日益重要起来。高速公路作为我国 基础设施建设的重要组成部分, 在社会经济发展中占据 着举足轻重的地位。高速公路的成功修建有利于加快各 地区经济往来，加快各地区信息交流。高速公路建设所 需资金多，投资额大，如果单靠政府修建会导致政府出 现巨大的财政压力。这时, 一种新的合作模式 PPP (Public Private Partnerships) 出现, 政府和社会资本方 在一定时期内共同合作为公众提供公共产品和服务。
PPP 模式因其有利于减轻政府的财政压力、有利于提高 项目建设质量的优势，应用于基础设施——高速公路建 设中。然而在高速公路的修建过程中, 项目参与主体多、 建设周期长, 存在许多易发生的风险。因此, 对 PPP 项 目风险的控制决定着 PPP 项目的成败。对高速公路 PPP 项目风险研究有利于规避风险, 降低风险的发生。

学者们对 PPP 项目风险进行了许多研究。在定性研 究方面, 开霞,柯永建等 (2009) 对中国 PPP 项目存在 问题的案例与项目失败或终止的案例进行归纳分析, 对 其失败的原因进行深入研究，找出影响项目失败的主要 风险因素, 最后提出了防范风险的建议与措施 ${ }^{[1]}$ 。Cheng Chen, Michael (2012) 等认为从项目参与的主体政府 方和社会资本方来看, 风险的分配存在着偏差, 高速公 
路 PPP 项目的风险分配偏向于实力强的一方, 风险的合 理分配可以减小项目双方权利不对等而导致的损失 ${ }^{[2]}$ 。 Albert P.C. Chan 等 (2012) 采用问卷调查的形式, 找出 影响高速公路项目的十大风险因素, 并对这些风险因素 按照发生的可能性大小进行排序 ${ }^{[3]}$ 。赵署蓉（2018）识 别了一带一路国家 PPP 项目基础建设过程中的风险, 基 于利益相关者共赢的视角提出了防范风险的建议 ${ }^{[4]}$ 。廖 石云（2019）基于我国跨境的 PPP 项目，分析了跨境项 目所面临的主要风险, 并提出了防范风险的对策建议 ${ }^{[5]}$ 。

在定量的角度方面, 在对 PPP 项目风险评价模型运 用上, 郭建 (2013) 认为公路由于未来交通流量的不确 定性, 公路 PPP 项目的风险分担问题是热点和难点问题, 采用了实物期权方法研究了不确定条件下延迟投资决 策对公路 PPP 项目的价值产生的影响, 并基于政府双边 保证的交通量分析了风险分担策略 ${ }^{[6]}$ 。刘秦南, 王艳伟 等 (2017) 运用系统动力学方法分析 PPP 项目运营风险 的动态变化, 识别了影响运营风险的因素并建立了存量 图, 根据熵值法确定变量的函数关系, 构建了 PPP 项目 运营风险的系统动力学模型并进行仿真模拟, 分析了 PPP 项目风险的熵值变化, 以提高对 PPP 项目运营风险 的控制与管理 ${ }^{[7]}$ 。Lakshyaet al. (2017) 通过对交通流、 工程造价等参数的识别, 结合印度的高速公路 PPP 项 目建立了判断项目盈利能力的 NPV-at-risk 模型, 用于 研究公路基础设施项目的财务风险, 并将模型应用于 30 个真实的 BOT 高速公路项目, 以识别关键风险并研究 风险防范策略 ${ }^{[8]}$ 。周盛世（2019）从银行、政府、建设 方三个主体角度分析了 PPP 项目的风险以及成因, 利用 Shapley 值法构建了多主体风险分担组合, 并对青岛地 铁进行了实证分析 ${ }^{[9]}$ 。项英辉 (2020) 在对基础设施 PPP 项目残值风险影响因素进行了分析并建立了风险评价 指标, 运用模糊物元评价模型对风险进行了评价 ${ }^{[10]}$ 。

总的来说, 学者们对 PPP 项目以及高速公路风险进 行了大量的研究, 而运用定量的方法对高速公路 PPP 项 目风险的研究较少。系统动力学 (System Dynamics, SD) 是一种专门研究系统中信息并将结果进行反馈的方法, 依据系统中的结果预测未来系统的行为并进行动态的 仿真分析, 关注系统内部因素的因果关系与变化过程, 在有限的信息条件下求解问题, 得出系统未来发展的趋 势和方向。为此, 通过引入系统动力学的方法对高速公 路 PPP 项目风险进行分析, 通过 Vensim 软件模拟风险 之间的变化并进行风险的仿真分析, 为高速公路 PPP 项 目风险管理提供支持。

\section{2.项目风险及其影响因素识别}

风险按不同的划分标准, 具有不同的风险分类。在 阅读了相关文献和书籍的基础上, 识别出影响高速公路 PPP 项目的主要风险包括: 政策风险、经济风险、金融 风险、建设风险、完工风险、运营风险、市场风险、环 保风险、不可抗力风险。

从项目外部风险划分为: 政策风险、经济风险、不 可抗力风险、环保风险。政策风险是指由于 PPP 项目法
律政策的变动、政府内部决策行为的不一致、政府审批 行为的不及时以及政府行为的临时改变给项目带来超 出预期的损失，从而导致项目的延期开工、甚至导致项 目失败。影响政策风险因素有政策稳定性、项目审批延 误、政府对项目的支持; 经济风险是由于受到国内宏观 经济形态的不利影响, 如国家经济发展形势下滑、通货 膨胀率等因素对项目的融资、项目资金流的运转产生影 响导致项目支付成本过高产生的风险。影响经济风险的 因素有经济发展水平、通货膨胀率; 环保风险是指高速 公路项目在建设和运营中会占用一定的自然资源, 对生 态环境产生不利的影响。一是对自然环境的破坏,造成水 土流失、自然植被破坏、占用了耕地等。二是对环境的 污染, 主要是行驶车辆所产生的噪声、废气、尘埃等注入 沿线环境使沿线环境质量降低。由于近年来响应国家加 强环保的号召, 履行环保责任, 恢复破坏的生态环境, 支出了大量的环保成本。影响环保风险的因素有环保法 规的变动、需履行的环保责任等; 不可抗力风险是指工 程建设方在发生前无法对其控制、事情发生时又无法合 理防范、事情发生后又无法回避或克服的事件或情况， 结果造成了财产损失和人员伤亡。如自然灾害：高温、 雨雪、地震、山体滑坡等的发生。如意外事件: 施工人 员安全状况。不可抗力容易造成工程事故、停工或损害, 导致建设工期延误、工程成本增加甚至项目终止的严重 后果。影响不可抗力的风险因素有自然灾害、突发事件。

从项目内部风险划分为: 金融风险、建设风险、完 工风险、运营风险、市场风险。金融风险由于国内利率 水平的波动对项目的利息和负债产生的影响，导致社会 资本方承担超出预期的利息与负债额，造成巨大的财务 压力进而影响项目的资金流，造成不足以支付项目的建 设而产生的风险。影响金融风险的因素有国内实际利率 水平、项目负债规模、项目融资成本; 建设风险是指项 目因项目建设成本超出预算额度, 项目未按照规定时间 竣工承担违约的责任或者竣工后无法达到正规标准而 赔偿损失所产生的亏损。影响建设风险的因素有能源和 原材料的供应、劳动力状况、经营管理水平; 完工风险 是指高速公路项目建设未能按照项目预期时间、预定标 准、预计成本而产生损失的可能性。它是项目进入建设 阶段后, 投资方直接面临的最大风险, 它决定着项目是否 能顺利运营,投资者是否能得到投资回报,债权人是否能 按期收回本息。影响完工风险的因素有项目建设延期、 项目建设成本、项目达不到标准; 运营风险指项目公司 在完成建设高速公路的目标, 将高速公路移交后, 在运 营期产生的风险。高速公路在运营中由于采用落后的管 理水平、政府方面的监察力度不够以及管理人员自身的 综合能力欠缺等会导致此路段的交通流量减少、维护费 用增加、利润整体水平变差, 致使总体收益不足。影响 运营风险的因素有交通流量、收费标准变化、运营维护 成本。市场风险更多来自附近其他线路的修建, 更多的 道路出行方式的选择导致高速公路的交通流量不足而 使项目运营公司产生巨大亏损。在运营期内, 高速公路 附近地区同时存在着其他出行方式与高速公路竞争, 导 致高速公路的车流量的分流, 影响了高速公路的预期收 益，甚至导致高速公路资金流的断裂。与此同时，受到 


\section{1 存量流量图}

风险。影响市场风险的因素有项目产品市场价格变动、 其他相关线路竞争。

\section{3.高速公路 PPP 项目系统动力模型建立与仿 真分析}

仿真分析是系统动力学模型重要的应用部分, 通过 动态模拟发现系统中存在的问题, 为规避风险提出对策 建议。从存量流量图中, 能直观地表达出各个变量之间 的逻辑关系与各个变量的性质, 具体如图 1 所示, 包括 23 个常数变量, 包括 9 个水平变量, 9 个速率变量。

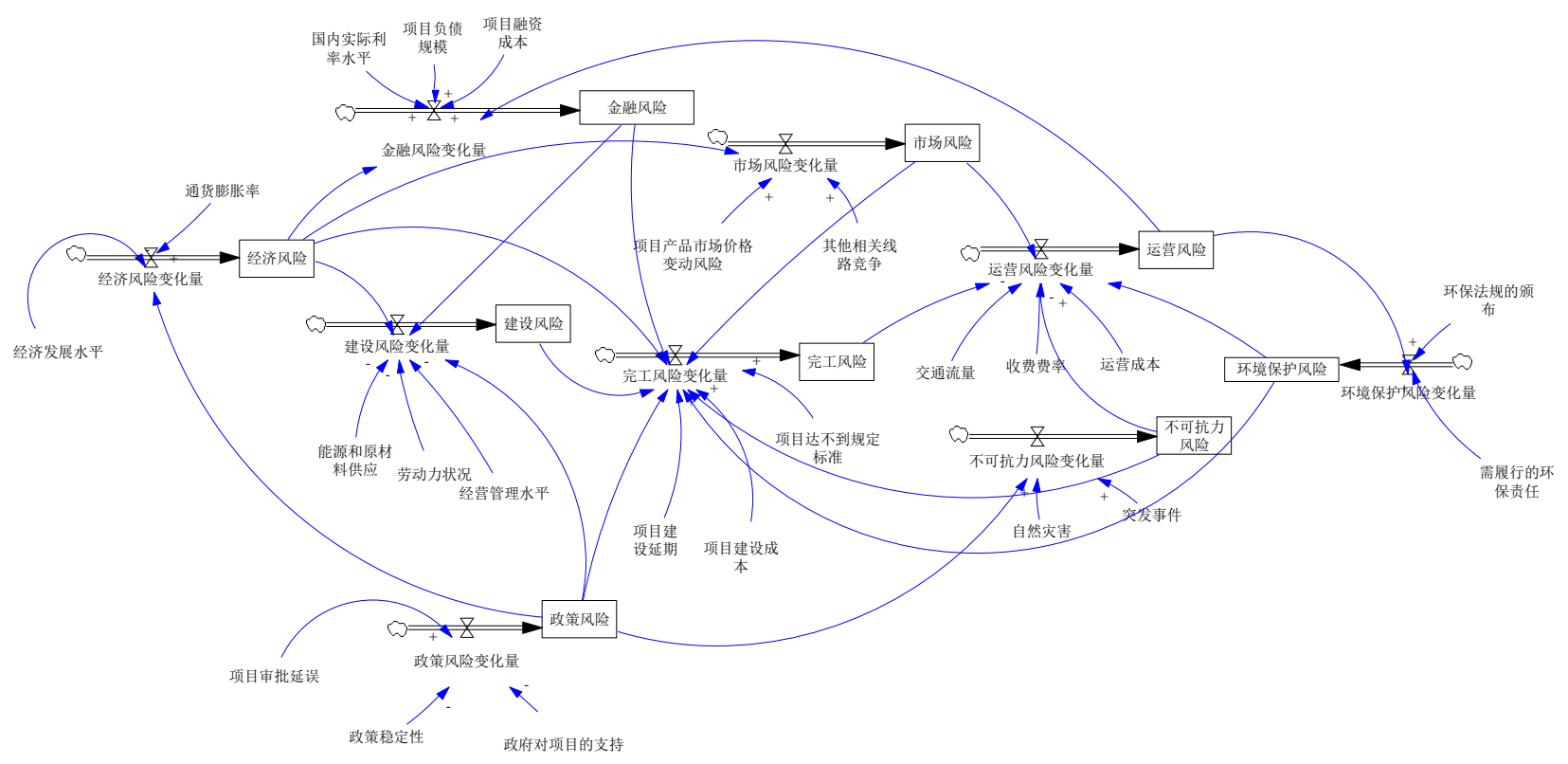

图 1 高速公路 PPP 项目风险存量流量图

\section{2 模型参数确定}

高速公路 PPP 项目系统边界点估计。本文采用专家 评分法对高速公路 PPP 项目进行评分获得风险数据。为 了保证专家打分结果的可比性和一致性以及计算结果 的统一性。参考相关研究, 使边界点的数值设定在 $0 \sim 1$ 范围内。0 表示该风险因素发生的可能性极小且几乎不 造成损失, 1 表示该风险因素发生的可能性极大且给系 统造成极为严重的损失。然后对不同风险因素每组数据 应用 $x i=a+4 m+b / 6$ 公式进行计算, 其中公式中 $a$ 代 表影响高速公路 PPP 项目的最小数值; b 代表影响高速 公路 PPP 项目最可能数值; c 表示影响高速公路 PPP 项目稳定最大数值。运用公式计算整理, 可以得到高速 公路风险变量的初始数。

主观赋权法带有评价者的主观意愿, 带有一定的主 观主义色彩, 缺少客观性。客观赋权法展示了准确的数 学方法, 具备一定的数学严密性, 但是缺少了评价者的
个人判断。将主观赋权方法和客观赋权方法相结合, 对 风险因素进行综合赋权，既避免了评价者的主观性又避 免了数学方法的单一性, 使评级结果具有现实意义。因 此, 在风险因素初始值确定的基础上, 运用综合赋权法 确定风险因素的权重。

\section{3 模型权重的确定}

选择了综合赋权法来确定权重。关系分析法的主观 赋权法其基本原理是：对评价指标按进行排序，确定它 们的序关系; 然后对排序之后的相邻指标间的重要程度 进行打分。最后, 对指标的打分结果进行一定的计算, 得到指标的主观权重。熵值法的客观赋权方法, 其中 心思想是指根据指标提供信息的大小来确定指标权重。 
融风险。运营风险是整个风险系统中最重要的风险。完

\section{4 建立系统动力学方程}

依照高速公路 PPP 项目存量流量图中变量之间的 因果关系, 对风险指标进行量化处理。首先确定了风险 变量以及变量之间的函数关系后, 将风险指标的数值及 其权重带入方程之后, 建立高速公路 PPP 项目的系统动 力学方程。

（1）经济风险 $=0.4 *$ 经济增长速度 +0.32 *通货膨胀率

(2) 政策风险 $=0.32 *$ 政府对项目的支持 $+0.3 *$ 政策稳定 性 $+0.4 *$ 项目审批延误

(3) 金融风险 $=0.47 *$ 国内实际利率水平 $+0.14 *$ 经济风险 $+0.09 *$ 运营风险 $+0.27 *$ 项目融资成本 $+0.27 *$ 项目负债规 模

（4）建设风险 $=0.31 *$ 劳动力状况 $+0.17 *$ 政策风险 $+0.14 *$ 经济风险 +0.26 *经营管理水平 $+0.44 *$ 能源和原材料供应 $+0.13 *$ 金融风险

（5） 完工风险 $=$ Ln $($ INTEG $(0.07 *$ 不可抗力风险 $+0.09 *$ 市场风险 +0.11 *建设风险 $+0.17 *$ *政策风险 $+0.08 *$ *环境保 护风险 $+0.14 *$ 经济风险 $+0.13 *$ 金融风险 $+0.21 *$ 项目建设 延期 +0.35 *项目建设成本 +0.45 *项目达不到规定标准, 0$)$ ) 完工风险随着项目的建设逐渐增加, 但随着项目的完工, 后期的影响减弱, 故用 $\mathrm{Ln}$ 函数来表示。

（6）市场风险 $=0.31 *$ 劳动力状况 $+0.17 *$ 政策风险 $+0.14 *$ 经济风险 +0.26 *经营管理水平 $+0.44 *$ 能源和原材料供应 $+0.13 *$ 金融风险

（7）运营风险 $=0.07 *$ 不可抗力风险 $+0.36 *$ 交通流量 $+0.1 *$ 完工风险 $+0.09 *$ 市场风险 $+0.5 *$ 收费标准变化 $+0.08 *$ 环境保护风险 $+0.13 *$ 运营成本

（8）不可抗力风险 $=0.48 *$ 突发事件 $+0.52 *$ 自然灾害

（9）环境保护风险 $=0.52 *$ 需履行的环保责任 +0.48 环保 法规的颁布

\section{4.系统动力学模型仿真分析}

\section{1 模型仿真分析}

项目外部风险变化趋势：如图 2, 政策风险和经济 风险变化趋势接近于直线走势, 其风险变动趋势较为平 稳。政策风险的发生的可能性高于经济风险发生的可能 性。不可抗力风险、环保风险整体走势波动较小。虽然 不可抗力风险、环保风险在整个项目中属于发生概率相 对较低的风险, 但是一旦发生影响着高速公路 PPP 项目 的顺利完工。因此, 社会资本方应注重对自然资源、生 态环境进行保护，避免生产运营过程会产生较严重污染。 项目公司应提前了解高速公路的施工状况, 预防不可抗 力带来的风险。

项目内部风险变化趋势: 如图 2, 随着建设进程的 逐步推进, 建设时间的逐步增多, 项目内各个风险增长 趋势排序为: 运营风险增长幅度最大、完工风险次之、 建设风险较小, 市场风险在项目前期小于金融风险, 项 目后期随着项目完成建设, 市场风险随之增大并超过金
工风险走势是一条波动的上升曲线, 呈现逐步增大的趋 势, 这与项目建设的阶段完成情况是密切相关的。随着 项目建设的起步, 完工风险几乎为零。随着项目建设开 展, 项目面临的风险因素逐渐增大, 完工风险不断增大, 直至项目建设竣工期，完工风险波动趋势趋于平稳。

\section{2 敏感性分析}

敏感性检验是指在相同比例下改变模型中的某一 变量的数值大小，来观察模型运行结果的趋势图变化。 一般情况下, 模型中变量值的改变会影响输出结果的变 化, 但是不应发生走势图的大幅变化, 输出结果的图形 状态应与原变量图形形态是相一致的。如果变量值的改 变会造成模型走势图大幅度变化, 与原来的走势图产生 不一致的变化, 则在系统中该变量具有敏感性, 在实际 应用中存在着局限性。通过上文分析可知, 建设风险、 完工风险是高速公路 PPP 项目的关键风险。通过考察建 设风险、完工风险的变化趋势来检验高速公路 PPP 项目 系统动力学模型是否稳定。

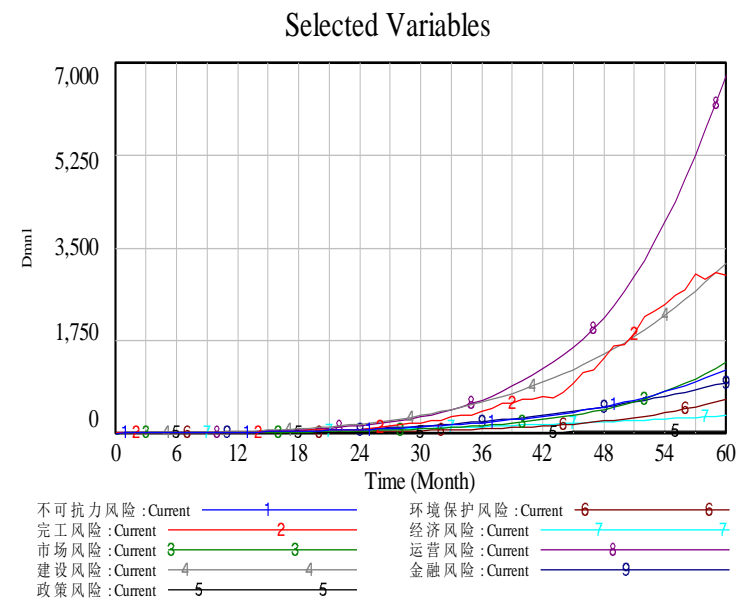

图 2 高速公路 PPP 项目风险仿真图

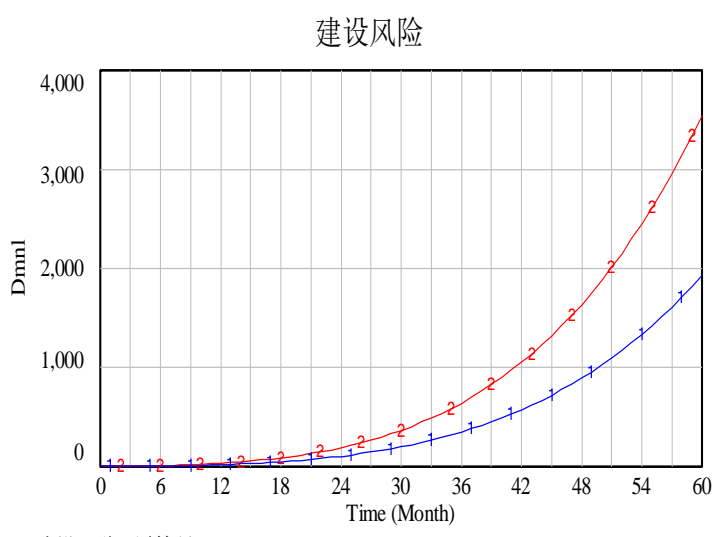

建设风险: 原情景

建设风险: 敏感度分析 \begin{tabular}{lllllllllllllllllllll}
\hline & 2 & 2 & 2 & 2 & 2 & 2 & 2 & 2 & 2 & 2 & 2
\end{tabular}

图 3 高速公路建设风险敏感性分析 
公路收费标准的变化。运营风险因素变化影响着运营风 险的变化, 交通流量数值提高 $10 \%$, 运营风险增强, 项 目公司应对交通流量的变化给予充分的重视并要预防 交通流量不足给高速公路的预期收入带来的影响。

第二，完工风险分析。由高速公路 PPP 项目风险仿 真图 1 可知, 完工风险是高速公路 PPP 项目需重点关注 的风险。完工风险影响着项目的顺利交付, 决定着项目 的成败。其中如图 6, 三类风险因素指标对完工风险的 影响程度有所不同，项目建设成本风险因素对完工风险 的影响最大, 项目建设延期风险因素对完工风险的影响 最弱, 项目建设成本超支风险因素对完工风险的影响处 于中间水平。因此，高速公路修建实施过程中，不同风 险因素的变化对完工风险的影响程度是不同的, 项目公 司应特别关注风险变化的原因并采取防范措施。在高速 公路修建过程中应严格控制项目建设成本, 其次需要保 证项目建设符合标准和项目如期完成。项目建设不符合 标准、项目建设延期等风险因素的变化同时也会增加系 统风险, 使完工风险系统不稳定。项目建设成本是重要 风险影响因素, 项目公司对成本费用进行监督, 以达到 降低成本, 提高收益的目的。

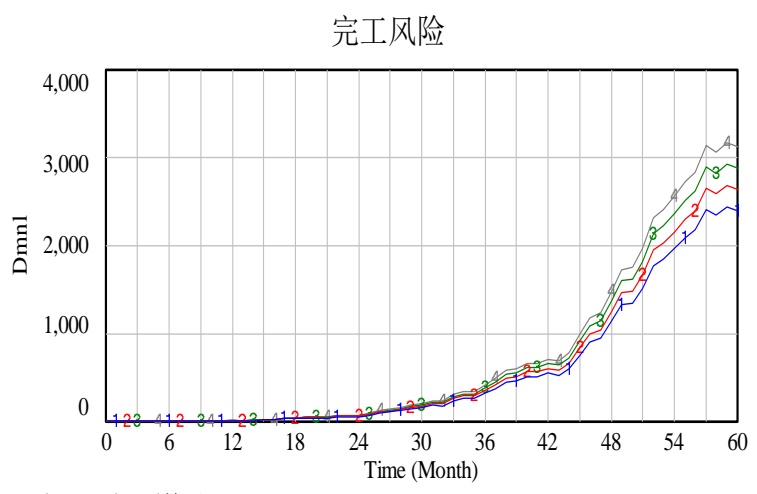

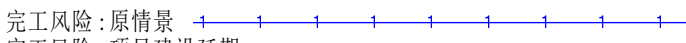

完工风险: 项目建设延期 $\begin{array}{lllllllll}-2 & 2 & 2 & 2 & 2 & 2 & 2 & 2\end{array}$ 完工风险: 项目达不到标准 $\begin{array}{llllllll}3 & 3 & 3 & 3 & 3 & 3 & 3 & 3\end{array}$ 完工风险: 项目建设成本增加

图 6 高速公路完工风险分析

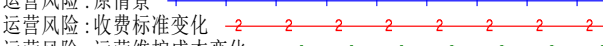
运营风险: 运营维护成本变化 $\begin{array}{llllllll}3 & 3 & 3 & 3 & 3 & 3\end{array}$ 营风险:交通施量变化

图 5 高速公路运营风险分析

\section{3 关键风险影响因素分析}

第一, 运营风险分析。在项目移交之后, 运营期是 项目的关键时期。运营风险在项目运营期是需要重点关 注的风险, 如图 5 在运营风险仿真图中, 描述了风险因 素变化时运营风险随时间的变化趋势, 将交通流量、运 营维护成本、收费标准风险因素数值同时提高 $10 \%$, 运 营风险在增强, 同时交通流量风险因素对运营风险的影 响大于运营维护成本风险因素对运营风险的影响大于 收费标准风险因素对运营风险的影响。因此在高速公路 PPP 项目运营期项目公司应密切关注项目交通流量的变 化并加以控制, 其次需要注意项目运营维护成本和高速

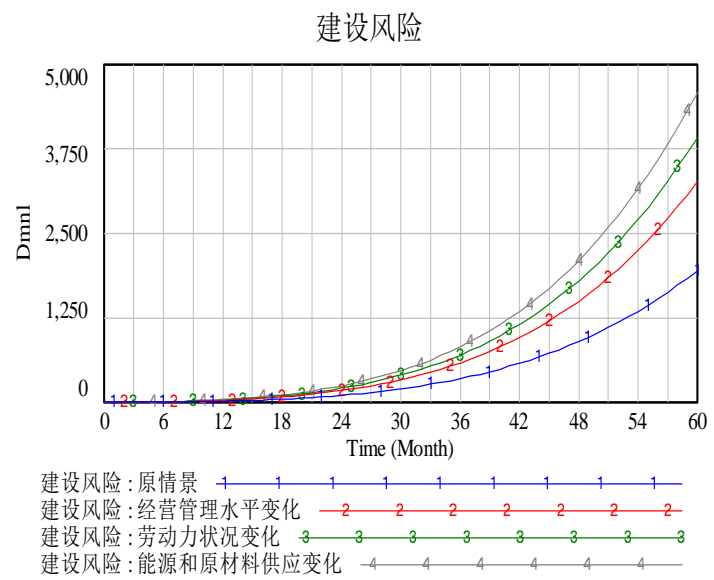

图 7 高速公路建设风险分析 
项目中的参与主体应树立风险管理的意识，优先关注发 生概率高的风险, 采取措施加强对高风险因素进行控制。 本文的不足之处在于研究数据来自于专家打分法, 变量值的赋值具有一定的主观性, 在某种程度上会影响 模型的仿真结果; 构建的系统动力学方程的复杂性还需 进一步提高, 加强对高速公路 PPP 项目风险系统的深入 研究。

\section{REFERENCES}

[1] Qi Xia, Ke Yongjian, Wang Shouqing. Analysis of main risk factors of China's PPP project based on case [J]. China soft science, 2009 (05): 107-113

[2] Cheng $\mathrm{C}$ hen, Michael Hubbard, Power relations and risk allocation in the governance of public private partnerships A case study from China[J].Seiverse Science Direct, 2012,31:39-49.

第一，做好风险预防工作。项目主体应对项目做充 分的准备, 选择具有项目建设经验的建造商进行合作; 项目主体应建立完善的风险准备预案工作, 在项目出现 成本超支、施工延期、不可抗力情况发生时, 有应对风 险措施的准备。

第二, 做好风险评估。项目公司应做好收入波动性、 项目风险评估，包括对收入波动性、成本构成、项目终 止的合同条款等认真评估; 项目公司应合理确定资本结 构安排, 要综合考虑成本、风险和权益等因素, 确定更 适宜项目特点的股权和债权结构; 项目公司还要认真评 估购买方的信用信誉情况，防止出现运营期违约风险。 其他需要认真考虑的因素还有项目建设方的财务实力、 项目相关方之间的关系、相关数量指标对风险的影响程 度。

第三，完善风险分担机制。结合高速公路项目面临 的风险的特点, 制定风险规避、风险转移等方案, 适当 地分散和化解项目风险, 防止出现无限责任; 树立系统 工程的观点。PPP 项目涉及众多参与者, 需要双方统一 思想, 共同参与, 遵守金融信用、商业信用和政务信用, 同时, 完善法律规则, 健全融资环境, 培育资本市场、 提升项目运作能力, 降低风险的发生。

\section{6.结论与研究不足}

通过本文研究可知, 高速公路 PPP 项目风险系统 中, 风险处于动态的变化过程中。风险不仅仅来源于项 目内部, 而且贯穿于高速公路修建的全过程, 与参与项 目的各方主体相关。因此, 高速公路的各个参与方, 要 以系统的视角认识和把握风险问题, 才能真正促进高速 公路项目在我国的有序、健康发展, 为解决其风险问题 提供有效途径。

有上文仿真分析可知, 风险因素与风险指标是正相 关关系。若控制影响风险的因素就可以减轻风险的发生。
[3] Esther Cheung, Albert P.C. C han, et al. A comparative study of critical success factors for public private partnerships(PPP)between Mainland China and the Hong Kong Special Administrative Region[J]. Emerald, 2012, 13: 647-666.

[4] Zhao Shurong, Yang Keke, and Lin Lin. One belt, one road,Risks and Countermeasures of PPP mode [J]. in area China administration, 2018 (11): one road infrastructure PPP project risks and Countermeasures [J]. building economy, 2019,40 (11): 9-13.

[6] Guo Jian. Study on traffic volume risk sharing strategy of Highway Infrastructure PPP project [J]. Management review, 2013,25 (07): 11-19 + 37

[7] Liu Qinnan, Wang Yanwei, Yao Ming Lai, Li Jing. Study on the evolution and Simulation of operation risk of PPP project based on system dynamics [J]. Journal of engineering management, 2017,31 (05): 57-61

[8] Lakshya Kumar, Apurva Jindal, Nagendra R. Velaga. Financial risk assessment and modelling of PPP based Indian highway infrastructure projects. Transport Policy, 2018, 62(2): 2-11.

[9] Zhou Shengshi, Zhang Ning, Zhang Xiaojuan. Risk sharing of metro construction PPP project based on Shapley value method $[\mathrm{J}]$. Journal of civil engineering and management, 2019,36 (06): 111-117

[10] Xiang Yinghui, Xing Yingnan, Zhao Yu. Residual value risk assessment of Infrastructure PPP project [J]. Construction technology, 2020,49 (03): 105-108.
[5] Liao Shiyun, Xiao Yan, Liu Zhangsheng.One belt, 